Tome 43, no 21. - Mai 1936.

\title{
Recherches expérimentales sur la détermination de l'embryon dans l'œuf de Sialis lutaria, Neuroptera
}

\author{
par
}

\section{Anne M. DU BOIS}

Bâle.

(Basler Stiftung für experimentelle Zoologie, Basel.)

Au cours du développement embryonnaire des Insectes, le stade blastoderme, c'est-à-dire le stade où l'œuf est constitué par une masse centrale de vitellus, entourée par une couche cellulaire unistratifiée, est immédiatement suivi par un stade, dans lequel les contours de la bandelette germinale deviennent visibles. Cette apparition de la bandelette, à la face ventrale de l'œuf, n'est pas due à une multiplication cellulaire intense dans cette région, mais est le résultat de la convergence d'un grand nombre de cellules blastodermiques dans cette zone. A la fin de ce processus, la bandelette germinale a acquis sa forme typique; elle est constituée par une couche de hautes cellules, serrées les unes contre les autres, tandis que tout le reste de l'œuf est limité par une membrane à cellules excessivement aplaties, le blastoderme extraembryonnaire. Quelle est la cause qui incite les cellules du blastoderme, primitivement toutes identiques, à se différencier ainsi, pour donner la première ébauche du futur embryon ? Les auteurs, qui ont attaqué ce problème expérimentalement, sont arrivés à des résultats fort différents, selon le groupe d'Insectes sur lequel ont porté leurs expériences.

Chez les Diptères, dans l'œuf fraîchement pondu, la masse centrale de vitellus, qui contient le noyau, est entourée par une couche

Rev. Suisse de Zool., T. 43, 1936. 
régulière de cytoplasme, le blastème germinal; la membrane vitelline et le chorion forment les enveloppes extérieures. Si, à ce stade, on détruit, par brûlure ou par ligature, une zone plus ou moins étendue de la partie antérieure, sans toucher au noyau, la partie postérieure est capable de se développer et se différencie en formant un embryon partiel postérieur, auquel manque une région, plus ou moins étendue, de la tête et du thorax. Inversément, si l'on détruit la partie postérieure, il y a formation d'un embryon partiel antérieur, auquel manque une partie de l'abdomen. Ces résultats, dus à Reith et à Pauli, prouvent que dans l'œuf de mouche, peu après la ponte, les différentes régions du futur embryon sont déjà toutes déterminées. Le blastème germinal est une mosaïque d'ébauches présomptives, qui ne deviendront visibles que beaucoup plus tard, au moment où les noyaux pénétreront dans le blastème. Les résultats des expériences de Hegner, sur des Coléoptères (Chrysomelidae), montrent que ces Insectes appartiennent également à ce type de détermination très précoce.

Chez les Odonates, la détermination de la bandelette embryonnaire s'accomplit selon un mode fort différent. Dans l'œuf de Libellule, au moment de la ponte, la masse de vitellus arrive au contact direct avec la membrane vitelline; il n'y a pas de blastème germinal. Ce n'est qu'au cours du huitième clivage, quand les noyaux arrivent à la périphérie de l'œuf, qu'un blastème se forme, par accumulation en surface, de toutes les petites masses cytoplasmiques, entourant les noyaux. La bandelette germinale, courte lors de son apparition, présente le phénomène de blastokinèse. A la suite de très grandes séries d'expériences, faites avec une précision remarquable, sur l'œuf de Platycnemis pennipens, SEIdel est arrivé à la conclusion qu'une région bien définie de l'œuf, correspondant à l'extrémité postérieure de la bandelette présomptive, agit comme centre de détermination. Toutes les fois que cette zone est gardée intacte, il y a différenciation d'une bandelette embryonnaire; toutes les fois que cette zone est détruite, il n'y a pas de différenciation. Ce centre de détermination agit au début du stade blastoderme; jusqu'à ce stade, l'œuf de Platycnemis est indéterminé. Pour que le centre puisse agir, il faut qu'il soit activé par la présence des noyaux; si l'on empêche expérimentalement les noyaux d'arriver en surface dans cette zone, il n'y a pas de différenciation de bandelette. 
Entre ces deux types extrêmes, à détermination précoce, en mosaïque, et à détermination tardive, sous l'action d'un centre, il doit exister toute une série de cas intermédiaires. Les résultats d'expériences ${ }^{1}$ faites sur l'œuf de Sialis lutaria montrent que ce Névroptère présente justement un de ces types intermédiaires.

Dans un œuf de Sialis, fraîchement pondu, il n'existe pas de blastème germinal. A partir du sixième clivage (environ 7-8 heures après la ponte, à une température de $19-20^{\circ}$ ), quand les noyaux sont encore à une certaine distance de la périphérie, un blastème se forme à la surface du vitellus (à la suite de mouvements dans la masse du vitellus ?). Au huitième clivage, les noyaux pénètrent dans ce blastème puis, à la suite de l'apparition des parois cellulaires, le blastoderme est constitué. La bandelette germinale est, dès son apparition - 20 heures environ après la ponte - assez longue et ne présente pas le phénomène de blastokinèse. Par ces deux derniers caractères, l'embryon de Sialis est très comparable à celui de diptère.

Si la détermination, chez Sialis, se fait selon le type précoce, en mosaïque, une ligature passant par le tiers antérieur de l'œuf doit détruire une partie de la tête, et avoir pour résultat, la formation d'un embryon partiel. L'expérience, réalisée sur des œufs, âgés de 4-6 heures, a pour résultat la différenciation, dans la partie postérieure, d'un embryon complet. Cet embryon est nain, mal proportionné, par suite du manque de place dans cette partie postérieure rétrécie, mais il présente une tête, pourvue de tous ses appendices. Ce résultat prouve que la détermination ne se fait pas selon le type précoce en mosaïque; jusqu'au sixième clivage, l'œuf de Sialis n'est pas déterminé.

Après des brûlures et des ligatures intéressant le tiers postérieur de l'œuf et pratiquées sur des œufs n'ayant pas dépassé le sixième clivage (œufs âgés de 1-6 heures), il n'y a jamais de différenciation d'une bandelette dans la partie antérieure. L'œuf reste vivant, les noyaux se divisent activement, mais sont incapables de former un

1 Les recherches expérimentales, relatées dans ce travail, ont été commencées en 1933, en collaboration avec le $\mathrm{D}^{\mathrm{r}} \mathrm{R}$. GEIGY, à l'instigation duquel elles sont dues. Les expériences de ligatures de 1934 ont été faites en commun par DuBois et Geigy. En 1935, les brûlures ont été réalisées par GEigy, les ligatures par DuBors. A la suite de circonstances indépendantes de ma volonté, j'ai dû terminer seule le travail, en me chargeant de l'étude complète, morphologique et histologique, du matériel et de la discussion des résultats. 
blastoderme normal. Ils arrivent par paquets irréguliers à la surface, les parois cellulaires ne se forment généralement pas. Après quatre ou cinq jours, ces œufs dégénèrent. Ce résultat est tout à fait analogue à celui obtenu par SEIDEL: la détermination de la partie antérieure dépend de l'intégrité de la partie postérieure. Il serait cependant trop hâtif d'en déduire que la Sialis rentre dans le type à détermination tardive, sous l'action d'un centre. Si l'on répète l'expérience, sur des œufs un peu plus âgés, chez lesquels, au moment de l'opération, le blastème cytoplasmique est constitué, mais où les noyaux ne sont pas encore arrivés en surface (œufs âgés de 7-9 heures) les résultats sont tout différents. Dans la partie antérieure, il y a différenciation d'un embryon partiel, qui se compose le plus souvent d'une tête très grande, ou d'un fragment de tête, plus rarement d'une tête complète et de un ou deux segments thoraciques. Ce résultat prouve que, dès le moment où le blastème apparaît à la surface de l'œuf, la bandelette embryonnaire existe à l'état présomptif; elle est complètement déterminée. Cette seconde série d'expériences, faite sur des œufs à blastème différencié, concorde en tous points avec les résultats des expériences chez les diptères. Le fait qu'il ne se forme qu'une tête, ou un fragment de tête dans la partie antérieure s'explique aisément par le fait, que la moitié environ de la bandelette primordiale représente à elle seule la future région céphalique. En avant de la brûlure ou de la ligature, il n'y a donc que du matériel déterminé à former la tête; ce matériel, ayant à sa disposition un espace beaucoup plus étendu que normalement, se développe en une tête, ou un fragment de tête, de dimensions exagérées.

Dans l'œuf de Sialis, au cours des clivages, il faut donc distinguer deux phases successives; dans une première phase, caractérisée par le manque de blastème, l'embryon n'est pas déterminé; dans une seconde phase, après l'établissement du blastème, l'embryon est complètement déterminé. Le moment où se fait la détermination doit donc coïncider avec le moment de l'apparition du blastème. En plus, c'est la partie postérieure de l'œuf qui joue le rôle principal dans le processus de la détermination. On peut émettre l'hypothèse que cette partie postérieure contient quelque chose d'analogue au centre de détermination découvert par SEIDEL; mais chez Platycnemis, le centre de détermination, pour pouvoir agir, doit être 
activé par la présence de noyaux, tandis que chez Sialis, la détermination s'établit sans que les noyaux interviennent, puisqu'au moment de la formation du blastème les noyaux n'ont pas encore atteint la périphérie de l'œuf.

L'œuf de Sialis, qui par ses caractères morphologiques: apparition tardive du blastème, longueur de la bandelette primitive, absence de blastokinèse, réalise un type d'œuf intermédiaire entre les deux types extrêmes cités au début, montre, dans le mécanisme de sa détermination, un compromis entre les mécanismes mis en évidence chez la Mouche, d'une part, chez la Libellule, d'autre part. 


\section{$2 \mathrm{BHL}$ Biodiversity Heritage Library}

Du Bois, Anne Marie. 1936. "Recherches expérimentales sur la détermination de l'embryon dans l'oeuf de Sialis lutaria, Neuroptera." Revue suisse de zoologie 43, 519-523. https://doi.org/10.5962/bhl.part.117686.

View This Item Online: https://www.biodiversitylibrary.org/item/148563

DOI: https://doi.org/10.5962/bhl.part.117686

Permalink: https://www.biodiversitylibrary.org/partpdf/117686

\section{Holding Institution}

American Museum of Natural History Library

\section{Sponsored by}

BHL-SIL-FEDLINK

\section{Copyright \& Reuse}

Copyright Status: In copyright. Digitized with the permission of the rights holder.

Rights Holder: Muséum d'histoire naturelle - Ville de Genève

This document was created from content at the Biodiversity Heritage Library, the world's largest open access digital library for biodiversity literature and archives. Visit BHL at https://www.biodiversitylibrary.org. 